\title{
Candidate locus analysis of familial ascending aortic aneurysms and dissections confirms the linkage to the chromosome 5q13-14 in Finnish families
}

Sakari Kakko, MD, PhDa

Tuija Räisänen, $\mathrm{MSc}^{\mathrm{a}}$

Minna Tamminen, MD, $\mathrm{PhD}^{\mathrm{a}}$

Juhani Airaksinen, MD, $\mathrm{PhD}^{\mathrm{b}}$

Kaj Groundstroem, MD, $\mathrm{PhD}^{\mathrm{C}}$

Tatu Juvonen, MD, $\mathrm{PhD}^{\mathrm{d}}$

Antti Ylitalo, MD, PhD

Paavo Uusimaa, MD, $\mathrm{PhD}^{\mathrm{f}}$

Markku J. Savolainen, $\mathrm{MD}, \mathrm{PhD}^{\mathrm{a}}$
From the Department of Internal Medicine and Biocenter Oulu, ${ }^{\mathrm{a}}$ University of Oulu, Oulu, Department of Internal Medicine, ${ }^{\text {b }}$ University of Turku, Turku, Department of Internal Medicine, ${ }^{c}$ Tampere University Hospital, Tampere, Department of Surgery, ${ }^{\mathrm{d}}$ University of Oulu, Oulu, Department of Internal Medicine, ${ }^{e}$ Central Hospital of Pori, Pori, and Division of Cardiology, ${ }^{\mathrm{f}}$ Department of Internal Medicine, University of Oulu, Oulu, Finland.

This study was supported by grants from the Research Council for Health of the Academy of Finland, the Finnish Foundation for Cardiovascular Research, the Sigrid Juselius Foundation and the Paavo Nurmi Foundation.

Received for publication March 1, 2002; revisions requested April 16, 2002; revisions received Aug 12, 2002; accepted for publication Aug 15, 2002.

Address for reprints: Tatu Juvonen, MD, $\mathrm{PhD}$, Department of Surgery, University of Oulu, PO Box 5000, FIN-90014, Oulu, Finland (E-mail: tatu.juvonen@oulu.fi).

J Thorac Cardiovasc Surg 2003;126:106-13

Copyright ( $\odot 2003$ by The American Association for Thoracic Surgery

$0022-5223 / 2003 \$ 30.00+0$

doi:10.1016/S0022-5223(03)00037-0
Objective: The purpose of the study was to carry out a candidate gene analysis in families with familial thoracic aortic aneurysms and dissections.

Methods: The study material consisted of 11 Finnish families (with 115 members genotyped) who underwent echocardiographic examination for measurement of the aortic root diameter. Selected candidate genes included the loci for Marfan and Ehlers-Danlos syndromes, the genes of matrix metalloproteinases 3 and 9 and tissue inhibitor of metalloproteinase 2 as well two loci on the chromosomes 5q13-14 and 11q23.2-q24, previously found to be linked to the disease.

Results: The chromosomal locus 5q13-14 was linked to the disease risk (nonparametric linkage score 3.0, $P=.005$ ) confirming the previous linkage. Other candidate genes and loci were excluded as major loci in these families.

Conclusions: The identification of the gene at chromosomal location 5q13-14 causing the development of such diseases would give us important knowledge on the pathogenesis of the disease and enable the identification of subjects at risk. This in turn would lead to appropriate treatment before the occurrence of fatal complications and, likely, to the development of new treatment methods.

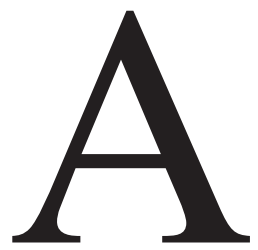

ortic aneurysm and dissections (including both abdominal and thoracic aorta) are responsible for about $1 \%-2 \%$ of the total mortality in affluent societies, aneurysm rupture being the first and often fatal manifestation of these diseases. The most important risk factors associated with the development of thoracic aortic aneurysms and dissections are chronic obstructive pulmonary disease, hypertension, and aging. At least one fifth of the aneurysms of the thoracic aorta other than those in patients with Marfan syndrome are due to genetic factors. ${ }^{1}$ The genetic risk has been illustrated in a family study, in which the risk of a brother of a patient with thoracic aortic aneurysm of developing an aneurysm was 11 times higher than the risk in the general population, whereas for sisters it was two times higher. ${ }^{2}$ In addition, familial cases are younger and their aneurysms grow faster than the sporadic ones. ${ }^{1}$

Although an obvious genetic risk on a proportion of patients with thoracic aortic aneurysms and dissections has been clearly defined, no major genetic locus for familial thoracic aortic aneurysms and dissections (FTAAD) has been found at the 
population level so far. The inheritance pattern of FTAAD has been suggested to be autosomal dominant but complex with reduced and age-dependent penetrance. ${ }^{3}$ Other inheritance patterns have, however, also been proposed suggesting genetic heterogeneity. ${ }^{1,2}$ Although patients with Marfan syndrome and Ehlers-Danlos syndrome type IV have an increased risk for the development of aortic aneurysm and dissections, only $<10 \%$ of patient with thoracic aortic aneurysm have Marfan syndrome and even fewer have EhlersDanlos syndrome. ${ }^{1}$ The loci for such rare syndromes have been mapped. The major locus for Marfan syndrome is located on the chromosome 15q21.1 (fibrillin 1, FBN1) and the second potential, still unconfirmed, locus on the chromosome 3p24-p25, whereas Ehlers-Danlos syndrome type IV is due to mutations of the type III collagen gene (COL3A1) on the chromosomal location $2 \mathrm{q} 31 .{ }^{4-7}$ These loci have also been found in some families to be linked to the development of aortic aneurysms without any other manifestations typical of such syndromes. ${ }^{6,7}$ Recently, two genome-wide linkage studies have found two novel loci for FTAAD in the chromosomal locations 5q13-14 and 11q23.2-q24. ${ }^{8,9}$ Among patients with abdominal aortic aneurysms, an association has been found between the risk of developing the disease and either genetic polymorphisms or the level of gene expression for matrix metalloproteinases 3 and 9 (MMP3 and MMP9) and tissue inhibitor of metalloproteinase 2 (TIMP2). ${ }^{10-12}$

The first clinical manifestation of FTAAD is often aortic rupture, which is frequently fatal. ${ }^{13}$ The identification of major loci and therefore the subjects at risk would make the treatment possible before such a severe complication occurs. The genetic homogeneity and small number of original founders make the Finnish population ideal for the disease gene identification by decreasing locus and allelic heterogeneity, not only in monogenic disorders, but also in more heterogeneous multifactorial disorders, like FTAAD. ${ }^{14}$ The aim of the current study was to test whether any of the candidate loci, including loci for 5q13-14, 11q23.2-q24 and FBN1, and the genes of COL3A1, MMP3, MMP9, and TIMP2, are linked to the risk of developing ascending aortic aneurysms or dissections (type A) in 11 Finnish families taken from a homogenous population.

\section{Methods}

\section{Subjects}

Families with members having had thoracic aortic aneurysm and dissections were collected based on probands treated at the University Hospitals of Oulu and Tampere. Families with at least 2 members with thoracic aortic aneurysms or dissections were collected. The total number of families included in the present study was 11 (with 115 subjects genotyped), 7 from Oulu and 4 from Tampere. The pedigrees are shown in Figure 1. A blood sample for DNA extraction and lipid measurement was obtained from every study subject. A physical examination was carried out excluding classic Marfan syndrome in the families. The information about medication, past medical history, alcohol consumption (recorded as grams of alcohol consumed in a week), smoking (recorded as nonsmoker or current smoker, pack-years of smoking, and the number of cigarettes smoked currently), and leisure activity were elicited with a questionnaire. Death certificates were provided by Statistics Finland and were used, if based on autopsy, to confirm the phenotypes of the deceased family members. For most of the unaffected family members, transthoracic echocardiography with aortic root measurements was done to confirm the phenotype. The study was approved by the Ethical Committees of the Universities of Oulu and Tampere, and all subjects gave written informed consent.

\section{Echocardiography}

Transthoracic echocardiography was performed with the study subject in the left lateral recumbent position using at the University of Tampere an Acuson 128/XP10 (Acuson, Mountain View, Calif) and at the University of Oulu a Hewlett-Packard Sonos 5500 echocardiographic system (Hewlett-Packard, Andover, Mass). Transthoracic scanning was performed either with a $2.5-\mathrm{MHz}$ or $3.5-\mathrm{MHz}$ probe from the parasternal, apical, and subcostal windows. The echocardiographic examinations were recorded on continuous videotape for off-line analysis system. Cardiac chamber diameters and wall thickness were measured from transthoracic 50 $\mathrm{mm} / \mathrm{s}$ M-mode recordings according to the recommendations of American Society of Echocardiography. ${ }^{15}$ The mean of at least 3 consecutive cardiac cycles was used. Normal values for aortic root were taken from the Framingham Heart Study material, and taking into account sex, height, and age of the subject. ${ }^{16}$

\section{Genotyping of Microsatellite Markers}

MTS1 and MTS2 microsatellite markers were selected for the major Marfan syndrome locus (FBN1), and the sequences of the primers were taken as published. ${ }^{17}$ The following microsatellite markers were selected and their sequences were taken from the Genome Database (www.gdb.org): D11S1924 and D11S1302 for the MMP3 gene, CLG4B and D20S888 for the MMP9 gene, D17S836 and D17S784 for the TIMP2 gene, D2S117 and COL3A18 for the COL3A1 gene. The selection of the markers was based on the distance $<4 \mathrm{cM}$ from the candidate locus and on the high level of heterozygosity. D5S253, D5S2029, D5S626 for the chromosomal locus 5q13-14 and D11S1341, D11S924 and D11S528 for the chromosomal locus 11q23.2-24 were most significantly linked markers previously ${ }^{8,9}$ and, therefore, selected. After positive results were found in chromosome 5, more markers were genotyped in that area, namely D5S2089, D5S1464, D5S641, D5S806, D5S1725, and D5S107.

Polymerase chain reactions (PCR) were carried out utilizing the BioMek1000 automatic pipettor (Beckman Instruments, Fullerton, Calif) in a total volume of $7.5 \mu \mathrm{L}$ containing $30 \mathrm{ng}$ of genomic DNA, $200 \mu \mathrm{M}$ of each dNTP, 1.3-2.3 pmol of each primer, 0.3 units of AmpliGold polymerase (Applied Biosystems, Foster City, Calif) and $1.5 \mathrm{mM} \mathrm{MgCl} 2$ in the buffer recommended by the manufacturer. PCR was carried out with a pre-PCR heat step of 12 minutes at $95^{\circ} \mathrm{C}$, followed by 10 cycles of 30 seconds at $94^{\circ} \mathrm{C}, 30$ seconds at $55^{\circ} \mathrm{C}-64^{\circ} \mathrm{C}$, and 45 seconds at $72^{\circ} \mathrm{C}$ and finally by 30 cycles of 30 seconds at $89^{\circ} \mathrm{C}, 30$ seconds at $55^{\circ} \mathrm{C}-64^{\circ} \mathrm{C}$ and 45 

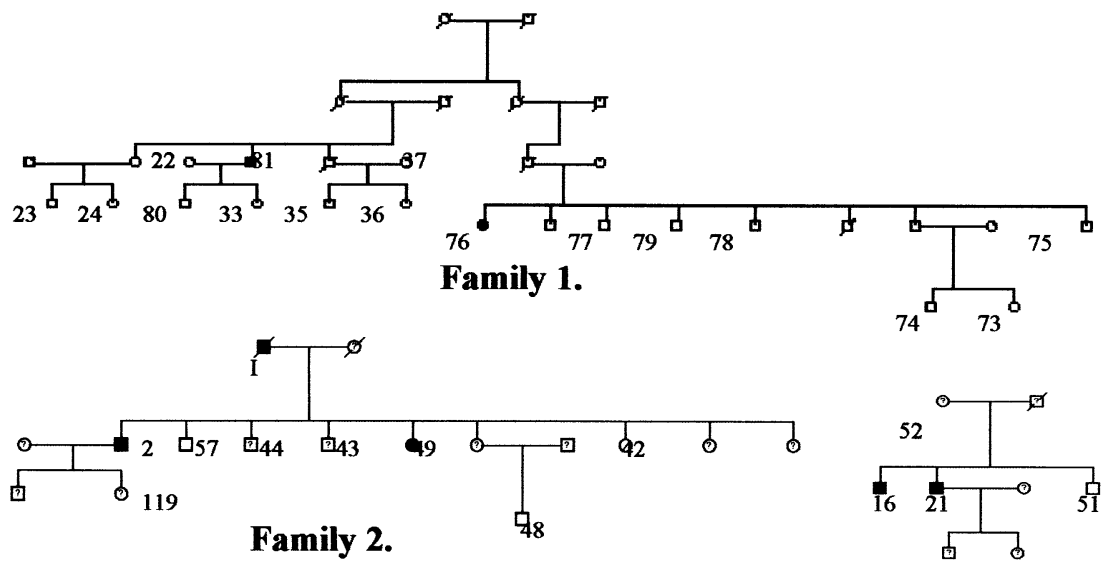

Family 3.
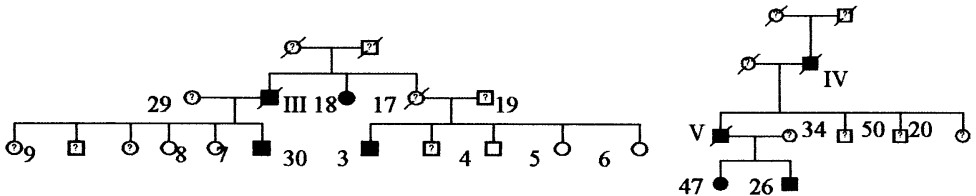

Family 4.

Family 5.

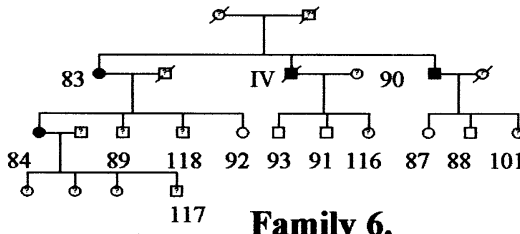

Family 6.

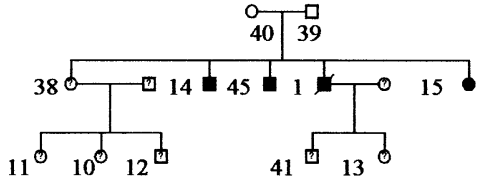

Family 11.

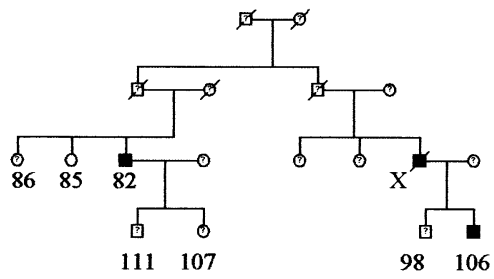

Family 7.

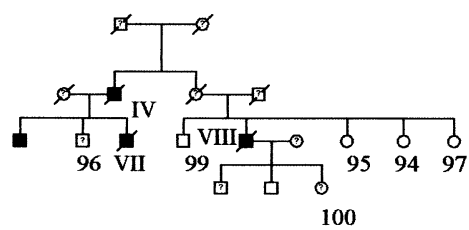

Family 9.

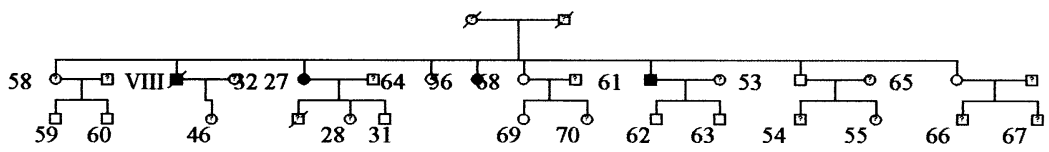

Family 8.

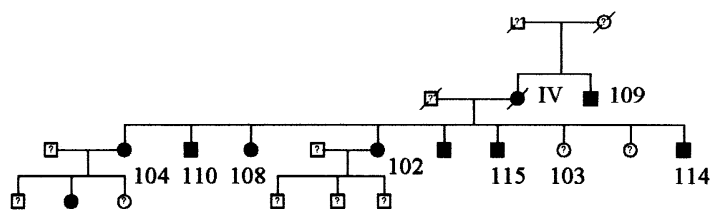

$105 \quad 121$

Family 10.

Figure 1. The pedigrees of the FTAAD families participating in the study. The study subjects genotyped are numbered with Arabic numbers, whereas family members not genotyped are numbered with Roman numbers. Three subjects with abdominal aortic aneurysms (subject $V$ in the family 5 , subject $X$ in the family 7 , and subject VIII in the family 8) were coded to have unknown phenotype in the analysis, but are marked as affected in the figure. 
TABLE 1. The manifestation of FTAAD in study subjects

\begin{tabular}{|c|c|c|c|c|}
\hline Family & Subject no. & Manifestation & Age at onset & Sex \\
\hline \multirow[t]{2}{*}{1} & 76 & Dilatation of ascending aorta (37 mm; $+4 \mathrm{~mm})$ & 56 & $\mathrm{~F}$ \\
\hline & 81 & Operated for aortic dissection (A-type) & 47 & $\mathrm{M}$ \\
\hline \multirow[t]{3}{*}{2} & 2 & Dilatation of aortic root $(51 \mathrm{~mm} ;+14 \mathrm{~mm})$ & 51 & M \\
\hline & 49 & Dilatation of aortic root (35 mm; $+4 \mathrm{~mm})$ & 40 & $\mathrm{~F}$ \\
\hline & 1 & Death due to aortic dissection (A-type) & 62 & M \\
\hline \multirow[t]{2}{*}{3} & 16 & Operated for aortic dissection (A-type) & 46 & M \\
\hline & 21 & Dilatation of aortic root (42 mm; $+5 \mathrm{~mm})$ & 46 & $\mathrm{M}$ \\
\hline \multirow[t]{4}{*}{4} & 3 & Dilatation of aortic root $(41 \mathrm{~mm} ;+4 \mathrm{~mm})$ & 38 & $\mathrm{M}$ \\
\hline & 18 & Dilatation of ascending aorta $(60 \mathrm{~mm})$, operated & 57 & $\mathrm{~F}$ \\
\hline & 30 & Operated for aortic dissection (A-type) & 28 & M \\
\hline & III & Death due to aortic dissection (A-type) & 53 & $\mathrm{M}$ \\
\hline \multirow[t]{3}{*}{5} & 26 & Operated for aortic dissection (A-type) & 26 & M \\
\hline & 47 & Dilatation of aortic root $(36 \mathrm{~mm} ;+4 \mathrm{~mm})$ & 29 & $\mathrm{~F}$ \\
\hline & IV & Death due to aortic dissection (A-type) & 65 & $\mathrm{M}$ \\
\hline \multirow[t]{4}{*}{6} & 83 & Dilatation of ascending aorta (42 mm; $+6 \mathrm{~mm})$ & 87 & $\mathrm{~F}$ \\
\hline & 84 & Dilatation of aortic root $(48 \mathrm{~mm} ;+16 \mathrm{~mm})$ & 40 & $\mathrm{~F}$ \\
\hline & 90 & Dilatation of aortic root $(44 \mathrm{~mm} ;+4 \mathrm{~mm})$ & 74 & M \\
\hline & IV & Death due to aortic dissection (A-type) & 62 & M \\
\hline \multirow[t]{2}{*}{7} & 82 & Operated for dilatation of ascending aorta & 64 & M \\
\hline & 106 & Dilatation of aortic root (39 mm; $+4 \mathrm{~mm})$ & 34 & M \\
\hline \multirow[t]{3}{*}{8} & 27 & Operated for aneurysm of ascending aorta $(65 \mathrm{~mm})$ & 56 & $\mathrm{~F}$ \\
\hline & 56 & Dilatation of ascending aorta $(40 \mathrm{~mm} ;+8 \mathrm{~mm})$ & 47 & $\mathrm{~F}$ \\
\hline & 61 & Dilatation of ascending aorta $(43 \mathrm{~mm} ;+6 \mathrm{~mm})$ & 54 & M \\
\hline \multirow[t]{3}{*}{9} & IV & Death due to aortic dissection (A-type) & 80 & $\mathrm{M}$ \\
\hline & VII & Death due to aortic dissection (A-type) & 52 & M \\
\hline & VIII & Death due to aortic dissection (A-type) & 72 & $\mathrm{M}$ \\
\hline \multirow[t]{9}{*}{10} & 102 & Dilatation of aortic root $(38 \mathrm{~mm} ;+5 \mathrm{~mm})$ & 46 & $\mathrm{~F}$ \\
\hline & 104 & Operated for annuloaortic ectasia & 50 & $\mathrm{~F}$ \\
\hline & 105 & Dilatation of aortic root $(40 \mathrm{~mm} ;+9 \mathrm{~mm})$ & 26 & $\mathrm{~F}$ \\
\hline & 108 & Dilatation of aortic root $(36 \mathrm{~mm} ;+4 \mathrm{~mm})$ & 47 & $\mathrm{~F}$ \\
\hline & 109 & Dilatation of aortic root $(45 \mathrm{~mm} ;+7 \mathrm{~mm})$ & 61 & M \\
\hline & 110 & Dilatation of aortic root $(44 \mathrm{~mm} ;+6 \mathrm{~mm})$ & 51 & $\mathrm{M}$ \\
\hline & 114 & Dilatation of aortic root $(50 \mathrm{~mm} ;+15 \mathrm{~mm})$ & 29 & M \\
\hline & 115 & Dilatation of aortic root $(47 \mathrm{~mm} ;+10 \mathrm{~mm})$ & 43 & $\mathrm{M}$ \\
\hline & IV & Death due to aortic dissection (A-type) & 53 & $\mathrm{~F}$ \\
\hline \multirow[t]{4}{*}{11} & 1 & Death due to aortic dissection (A-type) & 45 & $\mathrm{M}$ \\
\hline & 14 & Dilatation of ascending aorta $(40 \mathrm{~mm} ;+4 \mathrm{~mm})$ & 40 & M \\
\hline & 15 & Operated due to aneurysm of ascending aorta $(52 \mathrm{~mm})$ & 45 & $\mathrm{~F}$ \\
\hline & 45 & Operated for ascending aortic aneurysm & 29 & M \\
\hline
\end{tabular}

F, Female; $M$, male. The numbers of subjects and families refer to Figure 1. Figures in parenthesis show the aortic root diameter and how much it is over the upper normal limit. The upper normal limits for aortic root taking into account the age, height, and sex of the study subjects are taken from Vasan et al. ${ }^{16}$

seconds at $72^{\circ} \mathrm{C}$. PCR was completed with 10 minutes at $72^{\circ} \mathrm{C}$. The PCR products were electrophoresed with an ABI 377 automatic sequencer (Applied Biosystems). The size of the alleles was determined based on the Genescan-500 size standard (Applied Biosystems), which was included in every sample, and done by utilizing the Genescan and Genotyper programs (Versions 3.1 and 2.0, respectively; Applied Biosystems). The results of genotyping were confirmed by two independent observers.

\section{Statistical Analysis}

The phenotypes of patients younger than 26 years (the age of the youngest patients with aneurysm) were selected to be unknown to reduce the possibility of incorrect phenotyping due to possible age-dependent penetrance at the dominant model of parametric linkage analysis. Subjects with an aortic root $>3 \mathrm{~mm}$ over the upper normal limit or operated for aortic aneurysm or dissection were classified as affected. Those with an aortic root diameter smaller than the upper limit were classified as unaffected and the others as with unknown phenotype. Three subjects with abdominal aortic aneurysms (the subject $\mathrm{V}$ in the family 5 , the subject $\mathrm{X}$ in the family 7 and the subject VIII in the family 8) were coded to have unknown phenotype. The parametric and nonparametric multipoint linkage analysis was carried out with GeneHunter2 program $^{18}$ in the computers of CSC-Scientific Computing Ltd (Espoo, Finland). The test for genetic heterogeneity, ie, that different loci are linked to the FTAAD risk in different families, was carried out with the HOMOG program. The marker allele frequencies were estimated based on all founders and unrelated family members $(n=20)$. The 
TABLE 2. Characteristics of study subjects

\begin{tabular}{|c|c|c|c|c|}
\hline & \multicolumn{2}{|c|}{ Men } & \multicolumn{2}{|c|}{ Women } \\
\hline & Affected & Unaffected & Affected & Unaffected \\
\hline No. of subjects & 18 & 20 & 13 & 18 \\
\hline Age & $49.6[34-83]$ & $43.1[27-76]$ & $50.4[26-87]$ & $53.3[31-76]$ \\
\hline Total cholesterol (mmol/L) & $5.86(1.16)$ & $5.29(1.17)$ & $5.45(0.74)$ & $5.37(0.88)$ \\
\hline HDL cholesterol (mmol/L) & $1.20(0.31)$ & $1.26(0.32)$ & $1.58(0.45)$ & $1.73(0.42)$ \\
\hline LDL cholesterol (mmol/L) & $3.40(1.06)$ & $3.28(1.11)$ & $3.18(0.50)$ & $3.06(0.93)$ \\
\hline Triglycerides (mmol/L) & $3.78(7.34)$ & $1.64(0.98)$ & $1.49(0.69)$ & $1.20(0.50)$ \\
\hline Body mass index $\left(\mathrm{kg} / \mathrm{m}^{2}\right)$ & $26.9(3.3)$ & $26.5(4.2)$ & $23.0(3.0)$ & $25.0(4.9)$ \\
\hline \multirow[t]{2}{*}{ Aortic root diameter (mm) } & $\mathrm{n}=10$ & $\mathrm{n}=20$ & $\mathrm{n}=10$ & $\mathrm{n}=17$ \\
\hline & $43.6[39-50]$ & $33.5[27-38] \dagger$ & $42.1[35-65]$ & $29.4[24-33]^{*}$ \\
\hline Hypertension & $11(61 \%)$ & $5(25 \%)$ & $6(46 \%)$ & $3(17 \%)$ \\
\hline Coronary heart disease & $1(6 \%)$ & $1(5 \%)$ & 0 & $2(11 \%)$ \\
\hline
\end{tabular}

Figures are shown as number of subjects (percentages) or as means (standard deviations) or as means [ranges]. HDL, High-density lipoproteins. LDL, low-density lipoproteins. Subjects with unknown phenotype $(n=46)$ are excluded from the table.

${ }^{*} P<.01$.

$\dagger P<.001$

distances between the microsatellite markers and candidate genes were taken from GeneMap99 (www.ncbi.nlm.nih.gov/genemap) or from the Ensembl Genome Server (www.ensembl.org).

The NPL (nonparametric linkage) score was taken as a primary result since no assumptions of inheritance patterns (of the frequency of disease-causing allele in the population and of penetrances, ie, the probability of being affected, of different genotypes) were needed as in parametric linkage analysis. The NPL statistics measures allele sharing among affected individuals within a pedigree using all available marker data on chromosome at the same analysis and estimates the statistical significance for the detected linkage between marker loci and disease locus at the sample. For parametric linkage analysis, the selection of best models was based on the simulations made with the data and the dominant model was chosen (with the penetrances of the wild type, heterozygous and homozygous genotypes of 0.01, 0.90, and 0.90). In addition, the model with age-dependent penetrances (with the penetrances of $0.001,0.10$, and 0.10 for subjects younger than 30 years; $0.01,0.30$, and 0.30 for subjects between 30 and 40 years, $0.01,0.70$, and 0.70 for subjects $40-60$ years; and $0.01,0.90$, and 0.90 for subjects over 60 years, similar to that published earlier ${ }^{8}$ ) was used in the analysis. The frequency of the disease allele was assumed to be $0.1 \%$. However, based on simulations, the analysis was not sensitive for the changes in the frequency of the disease allele. The criteria for significances were taken as published, in which a nominal $P$ value of 0.01 was required for the confirmation of linkage. ${ }^{19}$

\section{Results}

Among genotyped subjects over 26 years old, there were 38 unaffected subjects (all of them having had their aortic root measured by ultrasound) and 31 affected subjects (the manifestations of FTAAD for every affected study subjects and their deceased family members are shown on Table 1). The phenotypes of 46 subjects remained unknown. In 17 subjects the aortic root was not measured, whereas 19 subjects had the diameter of their aortic root close to upper normal limit. There were 10 subjects younger than 26 years (the age of youngest patients), therefore, having their phenotype coded as unknown. In overall, the aortic root was measured for 85 subjects and ranged between 22 and $65 \mathrm{~mm}$.

The characteristics of the affected and unaffected study subjects excluding subjects with unknown phenotype are shown on Table 2. Among unaffected subjects, the aortic root was larger in men than in women (mean of 33.0 vs. $28.5 \mathrm{~mm}, P<.001$ ). Coronary heart disease was uncommon in the study subjects (Table 2) and there was no difference in the history of coronary heart disease between unaffected and affected subjects. However, the affected subjects had more frequently hypertension than unaffected subjects (55\% vs. $21 \%, P=.008)$. No significant differences in the lipid values or in body mass index were detected between affected and unaffected subjects. There were 13 women and 18 men in the affected subjects group.

The best multipoint nonparametric linkage (NPL) and logarithm of odds (LOD) scores for the genotyped loci at the initial screen are shown on Table 3. The genotyped loci, except the chromosomal locus 5q13-14, were excluded as a major locus for FTAAD. For chromosomal locus 5q13-14 more markers were genotyped yielding the best NPL score of $3.0(P=.005)$ at map position $+20 \mathrm{cM}$ (Figure 2) with the best parametric multipoint LOD scores of 2.01 (dominant model, Figure 2) and 1.10 (age-dependent penetrance model). Table 4 shows the best multipoint NPL scores and LOD scores for single families at the position with the highest overall scores showing that most but not all families were linked to the locus. The most significant single point NPL score, $2.48(P=.01)$, and the best parametric LOD score with dominant model, 3.16, were for the marker D5S1464. There was, however, no evidence for the locus heterogeneity between the families tested by HOMOG pro- 


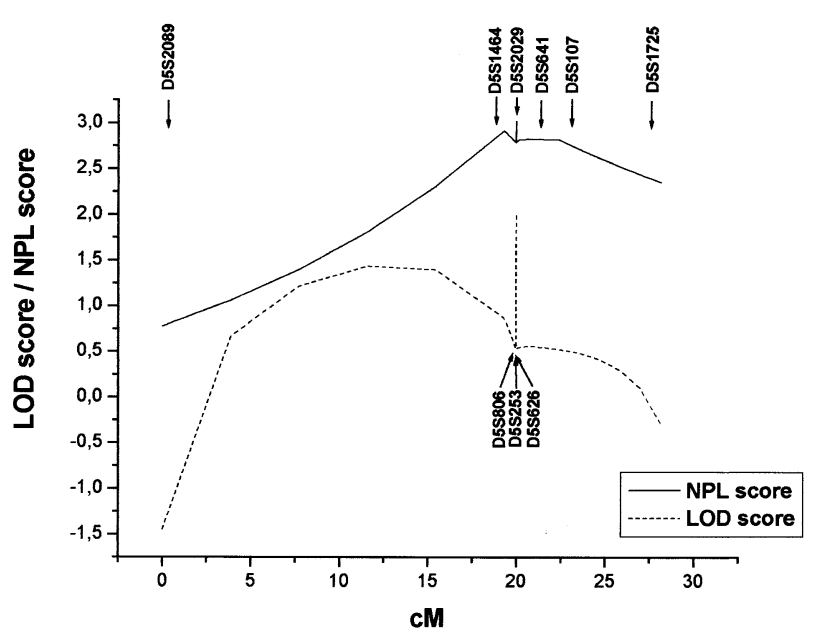

Figure 2. Multipoint NPL scores and LOD scores of dominant model for the chromosomal locus 5q13-14.

gram with the marker D5S1464, probably due to low number of the families. The haplotypes of markers at chromosomal location 5q13-14 for two families with the highest NPL scores (families 4 and 7) are shown on Figure 3.

\section{Discussion}

The risk of developing aneurysms and dissections of thoracic aorta is partly inherited. ${ }^{1}$ However, previous studies reported controversial results of the inheritance pattern and the major loci of FTAAD suggesting heterogeneous genetic background. ${ }^{1-3,6-9}$ The Finnish population, with small numbers of original founders and minimal immigration during many centuries, should be powerful for the disease gene identification. ${ }^{14}$ The aim of the current study was to search FTAAD locus in Finnish families using linkage analysis of candidate genes and loci and, by this approach, we were able to confirm the previous linkage of FTAAD to 5q13$14 .^{8}$ The multipoint NPL score $3.0(P=.005)$ meets the requirement for the confirmed linkage proposed by Lander and Kruglyak. ${ }^{19}$

The establishment of $5 q 13-14$ as a locus for FTAAD makes the testing of disease risk possible in those families with FTAAD risk linked to the locus. The fact that other loci have also been found to be linked to the FTAAD risk in single families ${ }^{6,7,9}$ makes the estimation of the disease risk in individuals somewhat difficult and at least, populationbased. Most of our families (Table 4), however, were linked to the locus pointing out its importance in the Finnish population. Because the same locus was originally found to be linked to the risk of FTAAD in around half of the Caucasian families collected at least partly from the USA, ${ }^{8}$ the importance of the locus should not be unique for Finnish population. Anyway, not all families, even in our homoge-
TABLE 3. The highest multipoint NPL and LOD scores of markers close to selected candidate loci for FTAAD

\begin{tabular}{lccc}
\hline & & \multicolumn{2}{c}{ Parametric LOD score } \\
\cline { 3 - 4 } Candidate locus & NPL score & Dominant & $\begin{array}{c}\text { Age- } \\
\text { dependent }\end{array}$ \\
\hline MMP3 & $0.34(P=.34)$ & -2.74 & -1.35 \\
MMP9 & $1.40(P=.09)$ & -2.86 & -1.45 \\
TIMP2 & $-0.48(P=.67)$ & -3.01 & -2.78 \\
COL3A1 & $-0.06(P=.50)$ & -6.63 & -5.01 \\
FBN1 & $0.16(P=.41)$ & -6.51 & -6.08 \\
5q13-14 & $2.67(P=.009)$ & 1.45 & 1.25 \\
11q23.2-q24 & $0.22(P=.40)$ & -4.03 & -1.17
\end{tabular}

The highest multipoint NPL score and LOD scores at the interval between genotyped markers of each candidate locus are shown.

TABLE 4. The highest multipoint NPL scores and LOD scores for single families at the locus $5 q 13-14$

\begin{tabular}{rrrr}
\hline & & \multicolumn{2}{c}{ Parametric LOD score } \\
\cline { 3 - 4 } Family & & Dominant & $\begin{array}{r}\text { Age- } \\
\text { dependent }\end{array}$ \\
\hline 1 & NPL score & -0.15 & -0.14 \\
2 & -0.38 & 0.66 & 0.66 \\
3 & 1.34 & 0.25 & 0.25 \\
4 & 1.41 & 1.13 & 1.13 \\
5 & 2.10 & -1.00 & -1.02 \\
6 & -0.18 & 0.84 & 0.13 \\
7 & 0.74 & 0.71 & 0.46 \\
8 & 2.65 & 0.07 & 0.34 \\
9 & 0.82 & -0.13 & -0.13 \\
10 & -0.03 & -0.95 & -0.95 \\
11 & -0.15 & 0.58 & 0.37 \\
Total & 1.72 & 2.01 & 1.10 \\
\hline
\end{tabular}

The highest total NPL score and LOD scores with both models were at the position $+20 \mathrm{cM}$ (Figure 2) and the scores for single families are shown at this position.

nous sample, were linked to the 5q13-14 locus showing the heterogeneous genetic background of FTAAD.

There were a few old subjects carrying the disease allele without aortic root dilatation. The observed reduced penetrance of FTAAD genes has been supported by earlier studies. ${ }^{3}$ FTAAD in the sample seemed to manifest from aortic root dilatation to fatal aortic dissection (Table 1). It is possible that the final manifestations of FTAAD, even in families in which the disease risk is linked to a major locus, are affected also by other genes or environmental factors (such as the presence of hypertension), not only by a single predisposing locus (like 5q13-14). Aneurysms and dissections of abdominal aorta are strongly associated with atherosclerosis, ${ }^{20}$ whereas no association between FTAAD and coronary heart disease was detected in our study subjects, thus, suggesting that the genetic defect leading to the development of FTAAD is not related to development of 


\section{Family 4}
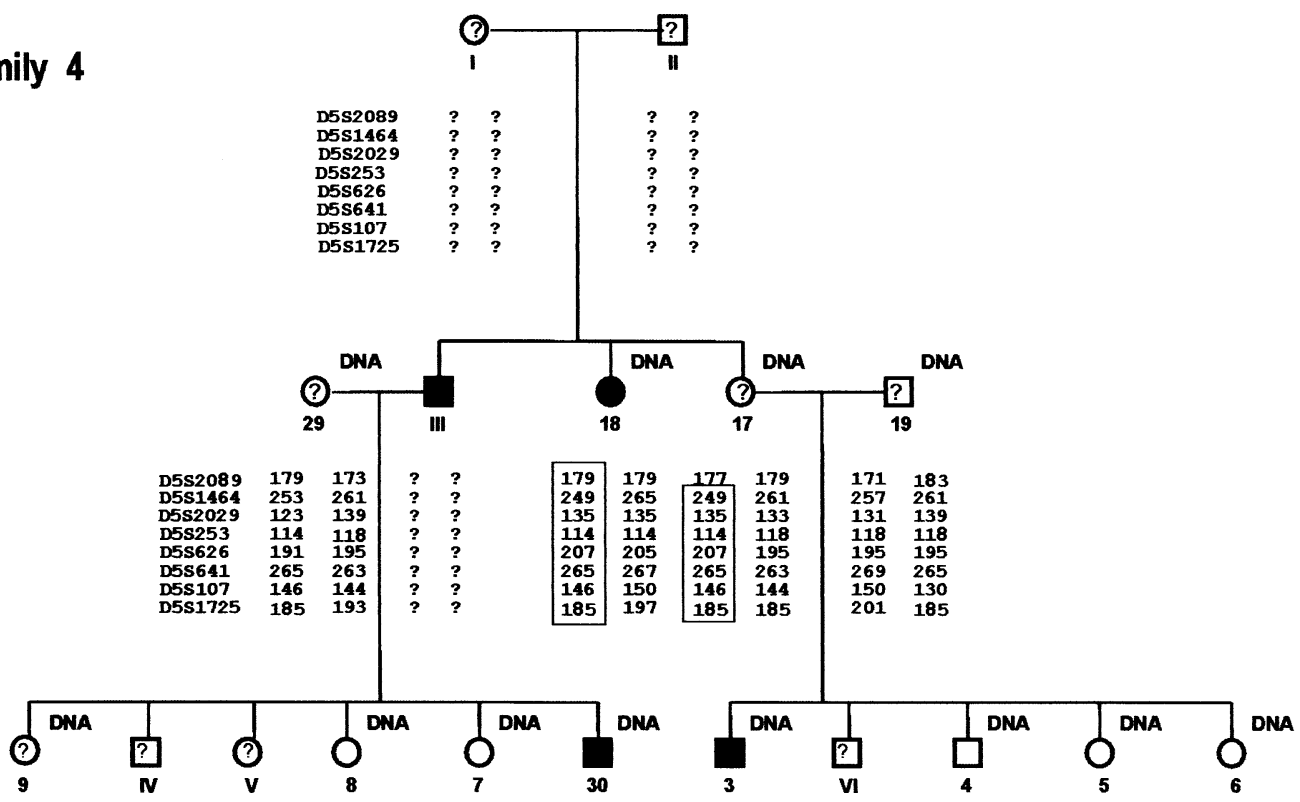

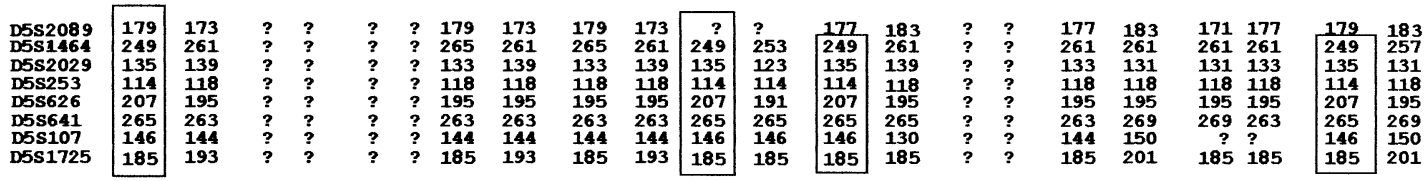

\section{Family 7}
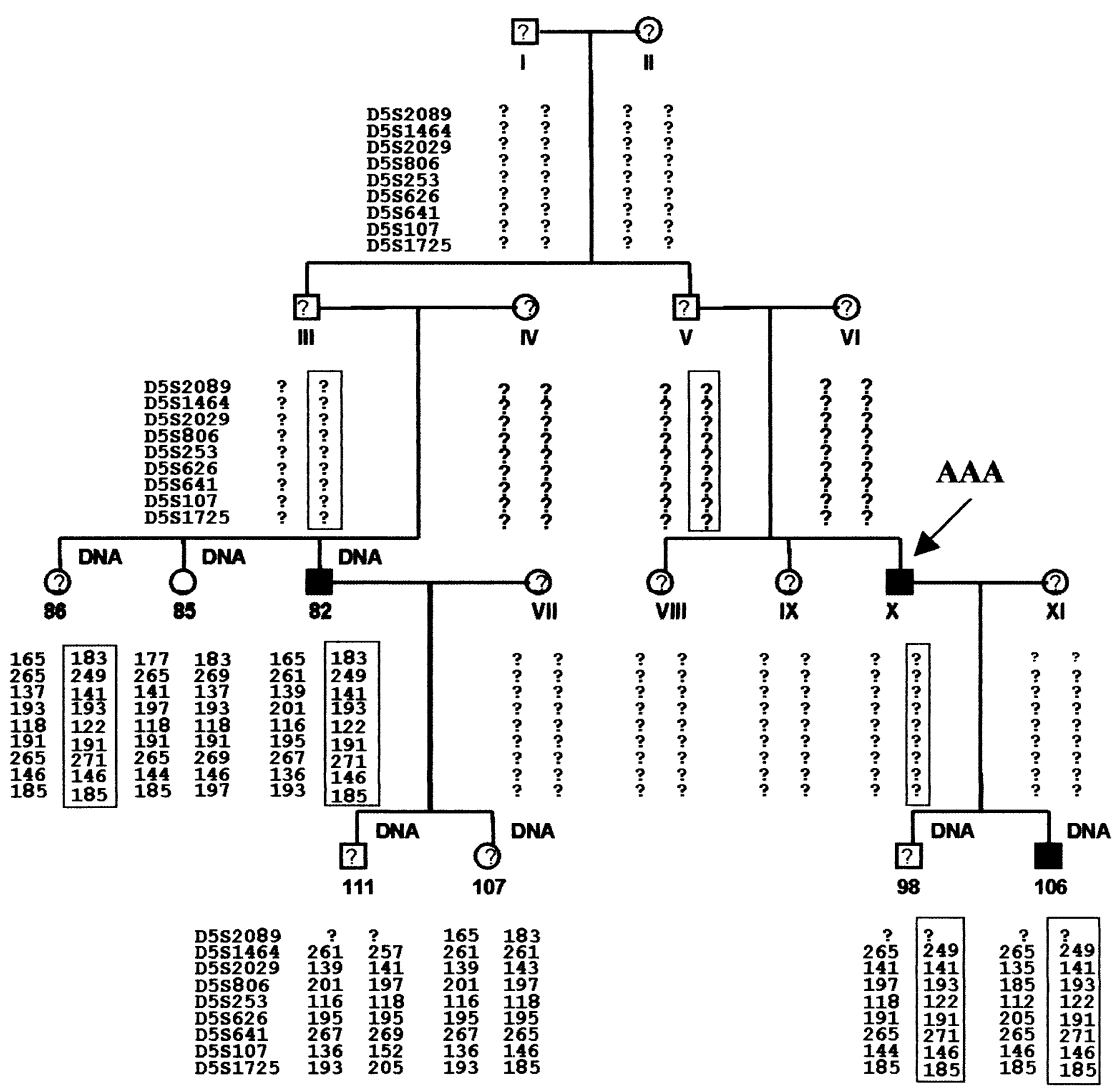

Figure 3. The haplotypes of markers at chromosomal location 5q13-14 for families 4 and 7 with the highest NPL scores. The subject $X$ in family 7 has abdominal aortic aneurysm (AAA). 
atherosclerosis. The affected subjects had more frequently drug-treated hypertension than unaffected subjects. However, such a difference might be overestimated, because antihypertensive treatment was easily started on at the time of diagnosis of aortic aneurysm or dissection.

The reliable and strict phenotyping of the study subjects enabled us to confirm the previous linkage in a sample consisting of only 11 families. Only those over 26 years of age and, by ultrasound measurements, determining their aortic root size to be smaller than the upper normal limit were considered as unaffected. The normal values were derived from the large Framingham study material, which took into account sex, age, and height. ${ }^{16}$ In addition to the patients operated on for aortic dilatation or aneurysms, those having their aortic root at least $3 \mathrm{~mm}$ over upper normal limit were considered as affected. The strict limit of $3 \mathrm{~mm}$ was taken to avoid incorrect phenotypes due to possible errors of measurements.

The next step will be to identify the disease gene and mutations in the gene enabling the development of new effective therapies preventing the development and progression of thoracic aortic aneurysms and dissections. Before such strategies would be available, identification of patients at risk for development of FTAAD would permit an adequate surveillance of such subjects and/or elective surgical repair before the occurrence of severe and often fatal complications.

The authors gratefully acknowledge the expert technical assistance of Saara Korhonen, Saija Kortetjärvi, Marja-Leena Kytökangas, Sari Pyrhönen, Sirpa Rannikko, and Eila Saarikoski. CSCScientific Computing Ltd is greatly acknowledged for providing us with the facilities for the high-performance computing. Dianna M. Milewicz, MD, PhD, Dongchuan Guo, PhD, and Zhengnan Yin, $\mathrm{PhD}$ are acknowledged for the help with the haplotype analysis.

\section{References}

1. Coady MA, Davies RR, Roberts M, Goldstein LJ, Rogalski MJ, Rizzo JA, et al. Familial patterns of thoracic aortic aneurysms. Arch Surg. 1999;134:361-7.

2. Biddinger A, Rocklin M, Coselli J, Milewicz DM. Familial thoracic aortic dilatations and dissections: a case control study. J Vasc Surg. 1997;25:506-11

3. Milewicz DM, Chen H, Park ES, Petty EM, Zaghi H, Shashidhar G, et al. Reduced penetrance and variable expressivity of familial thoracic aortic aneurysms/dissections. Am J Cardiol. 1998;82:474-9.

4. Dietz HC, Pyeritz RE. Mutations in the human gene for fibrillin-1
(FBN1) in the Marfan syndrome and related disorders. Hum Mol Genet. 1995;4:1799-809.

5. Collod G, Babron MC, Jondeau G, Coulon M, Weissenbach J, Dubourg $\mathrm{O}$, et al. A second locus for Marfan syndrome maps to chromosome 3p24.2-p25. Nat Genet. 1994;8:264-8.

6. Francke U, Berg MA, Tynan K, Brenn T, Liu W, Aoyama T, et al. A Gly1127Ser mutation in an EGF-like domain of the fibrillin-1 gene is a risk factor for ascending aortic aneurysm and dissection. Am J Hum Genet. 1995;56:1287-96.

7. Kontusaari S, Tromp G, Kuivaniemi H, Ladda RL, Prockop DJ. Inheritance of an RNA splicing mutation (G+ 1 IVS20) in the type III procollagen gene (COL3A1) in a family having aortic aneurysms and easy bruisability: phenotypic overlap between familial arterial aneurysms and Ehlers-Danlos syndrome type IV. Am J Hum Genet. 1990; 47:112-20.

8. Guo D, Hasham S, Kuang SQ, Vaughan CJ, Boerwinkle E, Chen H, et al. Familial thoracic aortic aneurysms and dissections: genetic heterogeneity with a major locus mapping to 5q13-14. Circulation. 2001; 103:2461-8.

9. Vaughan CJ, Casey M, He J, Veugelers M, Henderson K, Guo D, et al. Identification of a chromosome 11q23.2-q24 locus for familial aortic aneurysm disease, a genetically heterogeneous disorder. Circulation. 2001;103:2469-75.

10. Yoon S, Tromp G, Vongpunsawad S, Ronkainen A, Juvonen T, Kuivaniemi H. Genetic analysis of MMP3, MMP9, and PAI-1 in Finnish patients with abdominal aortic or intracranial aneurysms. Biochem Biophys Res Commun. 1999;265:563-8.

11. Yamashita A, Noma T, Nakazawa A, Saito S, Fujioka K, Zempo N, et al. Enhanced expression of matrix metalloproteinase-9 in abdominal aortic aneurysms. World J Surg. 2001;25:259-65.

12. Wang X, Tromp G, Cole CW, Verloes A, Sakalihasan N, Yoon S, et al. Analysis of coding sequences for tissue inhibitor of metalloproteinases 1 (TIMP1) and 2 (TIMP2) in patients with aneurysms. Matrix Biol. 1999;18:121-4.

13. Bickerstaff LK, Pairolero PC, Hollier LH, Melton LJ, Van Peenen HJ, Cherry KJ, et al. Thoracic aortic aneurysms: a population-based study. Surgery. 1982;92:1103-8.

14. Peltonen L, Jalanko A, Varilo T. Molecular genetics of the Finnish disease heritage. Hum Mol Genet. 1999;8:1913-23.

15. Henry WL, DeMaria A, Gramiak R, King DL, Kisslo JA, Popp RL, et al. Report of the American Society of Echocardiography Committee on nomenclature and standards in two-dimensional echocardiography. Circulation. 1980;62:212-5.

16. Vasan RS, Larson MG, Benjamin EJ, Levy D. Echocardiographic reference values for aortic root size: the Framingham Heart Study. J Am Soc Echocardiogr. 1995;8:793-800.

17. Pereira L, Levran O, Ramirez F, Lynch JR, Sykes B, Pyeritz RE, et al. A molecular approach to the stratification of cardiovascular risk in families with Marfan's syndrome. N Engl J Med. 1994;331:148-53.

18. Kruglyak L, Daly MJ, Reeve-Daly MP, Lander ES. Parametric and nonparametric linkage analysis: a unified multipoint approach. Am J Hum Genet. 1996;58:1347-63.

19. Lander E, Kruglyak L. Genetic dissection of complex traits: guidelines for interpreting and reporting linkage results. Nat Genet. 1995;11: 241-7.

20. Reed D, Reed C, Stemmermann G, Hayashi T. Are aortic aneurysms caused by atherosclerosis? Circulation. 1992;85:205-11. 